\title{
APORTES DE LA SOCIOLOGÍA AL ESTUDIO DE LA ALIMENTACIÓN FAMILIAR $^{1}$
}

\author{
SANDRA MILENA FRANCO PATIÑO
}

Manizales, 2010-04-30 (Rev. 2010-09-12)

\section{RESUMEN}

La alimentación es un fenómeno social y cultural, en tanto configura un escenario de interacción entre los sujetos, alrededor de circunstancias que entrelazan valoraciones culturales, significaciones subjetivas y relaciones sociales en tiempos y dinámicas particulares. En este trabajo se describen de manera sucinta los principales temas de investigación, objetivos, alcances y limitaciones de los estudios adelantados por las diversas disciplinas de la ciencia social, destacando de manera especial los significados sociales contenidos en las prácticas de alimentación familiar. Se reflexiona, además, sobre la importancia de los aportes de la sociología para la comprensión de los aspectos sociales y culturales del proceso alimentario, particularmente en el ámbito doméstico familiar, arista poco explorada desde las concepciones económicas y biológicas predominantes en el estudio de la alimentación.

PALABRAS CLAVE:

Alimentación, sociología, familia.

\section{CONTRIBUTIONS OF SOCIOLOGY TO FAMILY NUTRITION DYNAMICS}

\begin{abstract}
Nutrition is a docaial and cultural phenomenon since it constitutes an interaction scenery among individuals around circumstances which crosslink cultural evaluations, subjective significance and social relations in specific times and dynamics. This work briefly describes the most important research topics, objectives, scope and constraints of the studies carried out by various Social Sciences disciplines, especially highlighting the social meanings contained in the family nutrition dynamics. A reflection is also made about the importance of Sociology contributions to the understanding of the social and cultural aspects of the nutrition process, particularly in the family domestic sphere, a not widely explored side from the economic and biological conceptions predominant in the nutrition studies.
\end{abstract}

KEY WORDS: nutrition, sociology, family.

\section{INTRODUCCIÓN}

La alimentación es una construcción social y cultural. Los alimentos para ser susceptibles de consumo, pasan por un proceso de transformación que expresa 
normas culturales de clasificación y combinación. En éste proceso se convierten en productos deseables, es decir, en comida.

La comida no solo se usa para satisfacer la necesidad de alimentarse. A través de ella se aprehenden normas y conductas sociales mediante las cuales los individuos participan y se incorporan a la vida social (Mead, 1951; Simmel, 1986). La comida adquiere usos y significaciones diversas según el contexto histórico y social; no obstante, el proceso de socialización e interacción que ocurre en torno de la alimentación es el eje fundante del acto alimentario. El estar juntos, el encuentro regularizado y pautado respecto al proceso de alimentación, permite superar el nivel instintivo y fisiológico de satisfacer el hambre, para convertirse en un proceso socializador que supera la significación personal. De esta forma, el acto alimentario se conforma de un comensal, de comida y de situaciones sociales determinadas y específicas en las que se interrelacionan contexto y estructura, significaciones subjetivas y reglas objetivas.

Pese a que la alimentación es un proceso inherente a la existencia humana, no siempre ha sido considerada un hecho social objeto de conocimiento, debido a que su naturalización conduce a asumirla como parte de la vida cotidiana sin que requiera reflexiones ni cuestionamientos y a que, ante la inconmensurabilidad de los problemas sociales, solo se abordan aquellos que cobran relevancia o que se consideran problemáticos entre ciertas comunidades académicas, gremiales o comunitarias, que logran posicionar ciertos temas de interés en los ámbitos académicos y políticos.

Ante la complejidad del fenómeno, diversos autores (Díaz \& Gómez, 2001; Poulain \& Proença, 2003; Aguirre, 2004; Carrasco, 2007; Sanz, 2008; Vizcarra, 2008) coinciden en señalar la necesidad de adelantar estudios interdisciplinarios que permitan comprender los diversos ámbitos, dimensiones y aspectos que se conjugan en el proceso alimentario. Desde la interdisciplinariedad y la complementariedad de los métodos, tanto las ciencias naturales como las ciencias sociales empiezan a articular esfuerzos para enfrentar las diversas situaciones alimentarias de la sociedad moderna, y posibilitar un conocimiento integral de los fenómenos en estudio.

Este ensayo busca argumentar sobre la importancia de considerar un enfoque sociológico en la comprensión y análisis de las situaciones alimentarias, en especial aquellas que se desarrollan en el ámbito doméstico familiar, toda vez que las prácticas de alimentación expresan y configuran nuevas formas de ordenamiento social, que inciden en los comportamientos e interacciones de los sujetos, en el ámbito individual, familiar, comunitario y social.

Para cumplir con este objetivo, se presenta de manera general los principales temas de investigación, así como los objetivos, alcances y limitaciones de las investigaciones realizadas por las diversas disciplinas de las ciencias sociales que han estudiado el fenómeno alimentario en los últimos treinta años, período en que este campo ha logrado mayor relevancia ${ }^{3}$, para luego situarlo en el contexto colombiano. Seguidamente, se enfatiza en el enfoque sociológico, paradigma central para abordar los significados sociales de las prácticas de alimentación familiar. 


\section{LA ALIMENTACIÓN COMO OBJETO DE ESTUDIO EN LAS CIENCIAS SOCIALES}

Cuando se piensa en la alimentación y en sus diversos procesos constitutivos, se aprecia con claridad la necesidad de que diversas disciplinas participen en la comprensión y la resolución de los distintos problemas que van surgiendo al respecto. Sin embargo, esto no es suficiente para que la práctica científica asuma una orientación holística en el abordaje empírico de los hechos en estudio, dado que ésta obedece a marcos conceptuales que cada disciplina ha definido autónomamente, según los objetos de conocimiento que iluminan su ser y quehacer. Debido a ello, en pocos casos la alimentación se constituye en objeto de conocimiento ${ }^{4}$. Ésta aparece complementaria a otros problemas de investigación con enfoques teóricos y metodológicos diversos según las disciplinas que la estudie.

En las ciencias de la salud, existe una línea de investigación sobre trastornos del consumo alimentario como anorexia y bulimia; desde la perspectiva de la nutrición humana se evalúan los comportamientos alimentarios y la ingesta de calorías, vitaminas y minerales según los parámetros establecidos por sexo y edad (Moreno \& Galiano, 2006; Bejarano et al, 2009; Bravo, Cass \& Tranter, et al, 2008) ${ }^{5}$; igualmente, se estudian las deficiencias nutricionales de niños, niñas, adolescentes, madres gestantes y lactantes. Desde la perspectiva clínica, hay avances sobre la relación entre consumo de determinados alimentos y la aparición de algunas enfermedades.

Para las ciencias sociales, la alimentación como objeto de investigación presenta diversos focos de atención. La economía se ha centrado en identificar el consumo de alimentos y la modificación de éstos en el tiempo (Taren et al.; 1990; Booker, 1949; Morton et al., 2007; Prada, Herrán \& Ortiz, 2008). Para ello, se consideran las fuentes de recursos de los hogares, los ingresos de las personas y los presupuestos familiares que permiten acceder a la canasta básica de alimentos, pero se presta menor interés a los factores de desigualdad asociados a la distribución y el acceso a los recursos que viven las sociedades, los cuales inciden en las oportunidades para obtener alimentos suficientes y necesarios en el

mercado.

Las explicaciones se apoyan en información proveniente de registros oficiales ${ }^{6}$, que miden y cuantifican la compra de alimentos, el gasto promedio de los hogares en alimentación, el gasto en comidas fuera del hogar, tendencias del consumo, lugares preferentes de compra, etc.

Según Díaz y Gómez (2001), estas explicaciones presentan algunas limitaciones en cuanto a que: a) las categorías empleadas en los registros en su interés de generalización, homogenizan ciertos aspectos de la realidad, desconociendo la heterogeneidad del consumo en un mundo cada vez más diverso; b) las dimensiones valorativas, sociales y culturales en las que se inserta el fenómeno no son tenidas en cuenta, y tampoco se precisan las características del consumo; y c) las variables que utilizan los registros no corresponden con la realidad del ámbito doméstico y resultan insuficientes para evidenciar las diferencias que variables como género, edad, curso de vida, ciclo de vida familiar y condiciones de salud introducen en el consumo, y que, igualmente, son insuficientes para valorar y dimensionar la incidencia que tiene el consumo en la economía doméstica.

Otra línea económica ha visto el problema de la alimentación en relación con las condiciones de pobreza que viven los hogares, especialmente en los países en vía de desarrollo. Esta línea emergió a partir de la década del 70 del siglo XX, 
cuando el problema del hambre y las hambrunas se inserta en el escenario político y en las discusiones del desarrollo, momento en el cual se contempla la necesidad de que los gobiernos de las diferentes naciones intervengan sobre esta problemática en el mundo.

La Seguridad Alimentaria (SA) ha sido el enfoque utilizado para analizar los procesos de alimentación (producción, disponibilidad, consumo, distribución, calidad, inocuidad) en relación con la acción gubernamental; enfoque que ha transitado conceptualmente desde la oferta de alimentos, la capacidad de acceso de las personas y las familias a una canasta básica de alimentación, hasta la consideración de aspectos subjetivos y culturales que van más allá de la oferta y la demanda (Pérez de Armiño, s.f.; Pereira, 1999).

Al respecto, Amartya Sen (1981) desarrolla su teoría sobre titularidades al alimento, como explicación de las hambrunas. El autor plantea que la inseguridad alimentaria no depende tanto de la disponibilidad de alimentos en el mercado, sino del acceso efectivo que las personas y familias tienen a ellos, mediante lo que él denomina los derechos de acceso alimentario, los cuales dependen de las dotaciones iniciales que poseen las personas y las familias, y de las capacidades y recursos para acceder a los alimentos, bien sea a través del mercado, por ayudas del Estado, o por el intercambio de productos en la comunidad.

Los autores de esta corriente (Drèze \& Sen, 1989; Maluf, 1998; Parikh, 1992; Mellor, 1988) analizan el problema alimentario a la luz de las desigualdades sociales generadas por las economías de mercado y consideran que la pobreza, entendida no solo como la consecuencia de la concentración del ingreso, sino también como la falta de libertades y capacidades para acceder a mejores condiciones de vida, es uno de los factores de mayor incidencia en la inseguridad alimentaria.

Con base en este análisis, desde la década del 80 organismos multilaterales (Banco Mundial - BM, Fondo Monetario Internacional - FMI y la Organization for Agricultura and Food - FAO) han recomendado tomar medidas políticas ${ }^{7}$ orientadas a eliminar la pobreza, la inseguridad alimentaria $^{8} y$, con ella, el hambre que padecen las personas y las familias más vulnerables ${ }^{9}$.

Esta posición es criticada por algunos teóricos y organizaciones sociales en tanto la SA mantiene en el mercado el mecanismo básico de acceso a los alimentos, lo que excluye a la población de extrema pobreza que no logra insertarse en esta dinámica. Ante ello, plantean la SA, como marco desde el cual los países y las poblaciones puedan decidir con autonomía sus políticas de producción y abastecimiento, conforme a las prácticas y creencias culturales de los pueblos (Montagut \& Dogliotti, 2006).

El enfoque de la SA ha aportado al menos cuatro aspectos fundamentales para entender el problema alimentario: 1) la priorización de aspectos socioeconómicos en la explicación del hambre y las hambrunas; 2) la consideración de las situaciones específicas de vulnerabilidad que viven las familias y las personas en ésta; 3) la interrelación con otras esferas como el cuidado y la salud, y 4) la necesidad de contemplar el valor cultural del derecho a la alimentación y la percepción del riesgo de los afectados (Pérez de Armiño, s.f.).

La exploración de la influencia cultural de la alimentación y su relación con estructuras sociales ha sido abordada por la antropología. Desde la perspectiva estructuralista, se buscó entender las reglas y normas que subyacen al comportamiento alimentario, su evolución a lo largo del tiempo y en distintos tipos 
de sociedades, en los hábitos de presentación, preparación y elección de comidas (Díaz \& Gómez, 2001: 13). Los códigos y la red de significación en que se inscribe el acto alimentario expresan formas de organización de los pueblos en ciertos períodos históricos y los signos de distinción de las clases sociales (Bordieu: 1998).

En la línea antropológica del materialismo cultural, Harris (1989) plantea que en el comer interviene algo más que la pura fisiología de la digestión. La selección de los alimentos (de origen animal y vegetal), así como las preferencias alimentarias de un grupo determinado, están asociadas a lo que el autor denomina bueno o malo para comer. La determinación del consumo está en función del costo/beneficio, "ya que los alimentos preferidos reúnen en general más energía, proteínas, vitaminas y minerales por unidad que los evitados." (Harris, 1989: 14). El aporte de Harris a los estudios de la alimentación refiere a la interrelación existente entre naturaleza y cultura, toda vez que el proceso alimentario es resultado de las condiciones materiales de vida de una sociedad y de la forma como ésta manipula, controla y establece relaciones con factores condicionantes del contexto.

Posteriormente, con los desarrollos que alcanzó la antropología de la alimentación en Gran Bretaña y Estados Unidos (Carrasco, 2007: 88), el interés se concentró en observar las dinámicas alimentarias en el contexto de cambio social, cuyos resultados fueron de gran utilidad en la formulación de políticas gubernamentales y en el campo del desarrollo y el bienestar.

Una tendencia contemporánea de la antropología de la alimentación reconoce que los problemas alimentarios están en estrecha relación con el fundamento político e ideológico de la sociedad, materializados en la manera de identificar los problemas, las soluciones y las acciones para abordar las diversas situaciones alimentarias.

Al respecto, Carrasco indica que la antropología de la alimentación debe "entender los problemas alimentarios como resultado de la configuración de una estructura que somete a la población afectada a nuevas condiciones biológicas (desnutrido o mal nutridos) y nuevas condiciones sociales (dependientes, ayudados, subsidiados en la dimensión más básica del ser humano)" (2007: 94). Desde esta vertiente, las explicaciones del fenómeno alimentario identifican la incidencia de las acciones políticas de los países en las prácticas de consumo, en el manejo del entorno ambiental, en las condiciones para la productividad, la oferta y el acceso a los alimentos como garantía de derechos, y sitúan sus análisis en contextos de desarrollo sociopolíticos más amplios.

La sociología, por su parte, analiza la alimentación como una práctica social cotidiana que permite la supervivencia humana y la posibilidad de reproducción de las actividades sociales. En el contexto europeo, a partir de la década de los ochenta, se retoma la tradición desarrollada en Francia e Inglaterra sobre estudios de la alimentación, en una corriente de pensamiento denominada sociología de la alimentación, cuyo interés es la significación social de la alimentación, mediante el análisis de los patrones de consumo, las prácticas alimentarias en el contexto de sociedades modernas, las motivaciones y los factores culturales que inciden en el comportamiento alimentario, las desigualdades sociales expresadas en el acceso y consumo de alimentos, las relaciones de género y la distribución de poder en el acceso a recursos alimentarios y la incidencia de los factores sociales, familiares y culturales en las condiciones de salud. 
Algunos estudios han adoptado el contenido sociocultural constitutivo del acto alimentario, fundamentado por la sociología de la alimentación. Contreras y Espeist (2002) analizan las relaciones de reciprocidad familiar y social que permiten y sostienen la distribución y asignación de recursos alimentarios en adultos mayores. Rotenberg y De Vargas (2004) analizan los significados (percepciones, experiencias y valores) de las prácticas alimentarias durante el amamantamiento hasta la introducción de la alimentación cotidiana de la familia, en un barrio popular de Rio de Janeiro. Menasche, Marques y Zanneti (2008) indagan las representaciones de la producción y el consumo de alimentos entre agricultores y agricultoras de la región del Valle de Taquari (Río Grande del Sur, Brasil).

En esta línea interesa comprender los significados que construyen los sujetos a partir de su experiencia individual y familiar respecto a las prácticas de alimentación, así como también los procesos que ocurren al interior del ámbito familiar en torno a la alimentación, ámbito poco explorado pese a que social y culturalmente las familias se han concebido como el escenario fundamental para este tipo de actividades.

Otros estudios sociológicos resaltan los contenidos sociales de la alimentación desde la perspectiva de género, para visibilizar las diferencias del consumo alimentario entre clases sociales, entre grupos poblacionales o familias y grupos en circunstancias específicas (Grignon \& Grignon, 1980; Peréz Gil \& Díez Urdanivia, 2007). Asimismo, analizan las condiciones de inequidad y desigualdad social a las que ha estado sometida una gran parte de la población, mayoritariamente mujeres, en las políticas de combate a la pobreza y el hambre. Vizcarra (2008) analiza las condiciones de inequidad y desigualdad social a las que han estado sometidas una gran parte de las mujeres pobres de los países del hemisferio Sur ${ }^{10}$, a partir de la identificación del lugar que se les otorga en las políticas de combate a la pobreza y el hambre. Su análisis evidencia el desigual acceso al poder y a los recursos alimentarios que enfrentan las mujeres en sus propios hogares, la comunidad y el mercado para satisfacer la necesidad alimentaria de sus familias, en tanto las condiciones y las oportunidades para el acceso a recursos económicos, créditos, propiedad sobre la tierra y empleo son restringidos para las mujeres, lo que aumenta su vulnerabilidad a situaciones de pobreza. García et al. (2008) auscultan en la dinámica familiar aquellos aspectos relacionados con los hábitos alimentarios y las prácticas alimentarias de la vida moderna, para evidenciar cómo los cambios en los hábitos alimentarios se ven permeados por los cambios en el contexto social, además de las características socioculturales y económicas propias de las familias y los individuos que las conforman.

\section{LOS ESTUDIOS DE LA ALIMENTACIÓN EN EL CONTEXTO COLOMBIANO}

En el caso colombiano, la cocina, la mesa y los comportamientos alimentarios son temas recurrentes entre profesionales de la gastronomía, la nutrición y la historia. Los antropólogos y los sociólogos, a partir de los años 90 del siglo XX, empiezan a interesarse por los factores sociales y culturales que encierra la alimentación, la incidencia de lo cultural en las condiciones nutricionales de la población y, en especial, por explotar su potencial como categoría analítica para entender las formas de organización de la sociedad y sus relaciones políticas y sociales. 
Un acercamiento a los estudios sobre la alimentación en Colombia evidencia una preponderancia de investigaciones de carácter histórico orientadas a reconocer, principalmente, el origen y uso de los alimentos en el siglo XIX; la influencia que países como Inglaterra, Francia y España tuvieron en las costumbres culinarias (alimentos preferentes, utensilios, preparaciones, sabores, uso de aditivos y condimentos) en diversos períodos históricos del desarrollo de la sociedad y, en una perspectiva más contemporánea, describir y comprender las amplias y variadas costumbres manducatorias colombianas en el contexto de la diversidad cultural y regional que caracteriza al país.

Al respecto, Víctor Manuel Patiño (1984) ausculta la cultura material de los países de la zona ecuatorial, mediante la reconstrucción del origen y uso de las plantas y los alimentos durante la época prehispánica, el descubrimiento y la época contemporánea; asociado a los significados de los alimentos según características ceremoniales y la organización social. De manera similar, Aída Martínez (1985) en su volumen culinario mesa y cocina en el Siglo XIX, muestra el pasado gastronómico colombiano desde sus antecedentes aborígenes y coloniales hasta la entrada de platos y productos europeos. Su interés es conocer los cambios operados en las costumbres alimentarias de los colombianos $^{11}$ durante la independencia, cuando lejos de la intervención española, era necesario reconstruir de manera autónoma la vida del país. Como conclusión final, la autora plantea que, contrario a la tesis de que los cambios en las circunstancias económicas y sociales inciden velozmente en la alimentación, se constata el arraigamiento y la preservación que las culturas mantienen de los hábitos alimenticios, como uno de sus valores más estables.

En esta misma línea, Cecilia Restrepo (2008), en un estudio histórico en el Colegio Mayor de Nuestra Señora del Rosario en Santafé de Bogotá en los siglos XVIII y XIX, identifica los acontecimientos, las coyunturas políticas y las transformaciones históricas ocurridas en el período en estudio en la ciudad de Bogotá. Mediante el análisis del proceso alimentario ofrecido en dicha institución se puede determinar la posición social, las costumbres y los hábitos que afectan una cultura; de ahí que la autora describa las características de producción y abastecimiento, la preparación de alimentos, estructura del consumo y celebraciones, como estrategia para acceder a los hechos o fenómenos relevantes que configuraron a la ciudad.

El análisis histórico de los alimentos, los sabores, la forma de preparación y disposición de los mismos a lo largo del Siglo XIX, posibilita entender el porqué de ciertas costumbres y formas en las prácticas alimentarias actuales.

Además de la perspectiva histórica, otras investigaciones sobre la alimentación suelen ser complementarias a miradas económicas, nutricionales y productivas $^{12}$, como las tradiciones más predominantes y, en las últimas dos décadas, se posicionan investigaciones desde el enfoque de la Seguridad Alimentaria y Nutricional, sustento fundamental para la formulación y ejecución de la política pública en la materia y desde la sociología y la antropología de la alimentación.

En la perspectiva económica, las tendencias de consumo de alimentos han estado ligadas al estado nutricional de la población, en particular de niños, niñas, madres gestantes y lactantes. Al respecto, Álvarez y González (2002) estudiaron las prácticas alimentarias en familias rurales de la ciudad de Medellín (Antioquia), para identificar las preferencias en la decisión de compra y consumo de frutas y verduras, durante el período de gestación, lactancia e incorporación de alimentación complementaria de niños y niñas. Prada, Herrán y Ortiz (2008) 
analizaron el patrón alimentario y la capacidad de accesos a los alimentos de familias desplazadas en el municipio de Girón (Santander), beneficiarias de un programa de ayuda alimentaria, cuyos hallazgos indican que los bajos ingresos familiares, sumado al desconocimiento de alternativas nutricionales más ventajosas, constituyen los principales factores para la inseguridad alimentaria de esta población.

En otra dirección, desde el área de la salud y la nutrición se han realizado investigaciones orientadas a la identificación de cambios en los conocimientos, actitudes y prácticas alimentarias de pacientes diabéticos, con $\mathrm{VIH}$ y madres gestantes y lactantes. El interés de estos estudios se centra en el análisis de los cambios que genera la aparición de determinada enfermedad, los cuales están asociados ?generalmente? a modificaciones en los hábitos de alimentación. De igual forma, se indaga cada vez más en las creencias y los patrones alimentarios de las madres gestantes y lactantes, por la importancia que éstos tienen en el desarrollo infantil y por la primacía que las políticas de infancia otorgan a esta etapa del desarrollo.

El enfoque de la Seguridad Alimentaria y Nutricional ha sido fuertemente impulsado para el análisis de las políticas en esta materia. Absalón Machado (2003) en el campo de la economía aplicada ubica el problema alimentario en relación con factores estructurales que afectan la disponibilidad y acceso a los alimentos; en especial, ubica los cambios que ha vivido Colombia en los contextos internos y externos que moldean las políticas y la evolución del sistema agroalimentario y su problemática. Mantilla y Morales (2008) evalúan la política pública de la SA impulsada por el gobierno nacional desde el año 2005, con el propósito de establecer alcances y vacíos respecto al logro del derecho a la alimentación, como el más representativo de los derechos económicos, sociales y culturales DESC.

En una perspectiva antropológica, Mora de Jaramillo (1985) se propone entender el proceso de aculturación en el Amazonas colombiano, mediante el contacto y el proceso de intercambio de bienes alimentarios. La investigación, desarrollada con una perspectiva etnográfica durante los años 1974-1980, focaliza en el intercambio cultural entre indígenas y blancos ${ }^{13}$ en Leticia, para reconocer los alimentos que consumen o dejan de comer los grupos dominados (indígenas) y los alimentos de la región que aprenden a comer los blancos en ciertas circunstancias, como también las consecuencias que esto tiene en los cambios culturales en cada grupo.

De manera similar, y en el marco del rescate del legado étnico colombiano, Carlos Osorio (2002) estudió el patrimonio cultural y simbólico de los indígenas Paeces (Cauca) mediante la identificación de la racionalidad y las prácticas alimentarias de este grupo y los procesos económicos y tecnológicos asociados. El interés que orientó el trabajo etnográfico fue la comprensión de los elementos simbólicos que fundamentan "el arte de comer, entre los Paeces de Tierradentro", integrados a los componentes ambientales, económicos y tecnológicos que influyen en las prácticas, las creencias y las costumbres sobre la alimentación en este grupo étnico.

Estas investigaciones son un importante aporte desde la cultura alimentaria para dimensionar las características que fundamentan la dieta actual de los colombianos, tradición que se refleja en los gustos y prácticas de consumo. A su vez, pone de manifiesto la necesidad de ahondar en esta temática para reconocer las continuidades y las rupturas de las prácticas alimenticias en las que se expresan nuevas formas de organización y estructuración de la sociedad. 
Un análisis más sociológico explora el sistema de prácticas alimentarias cotidianas en familias de un barrio de clase media en la ciudad de Cali (Valle), para identificar las interacciones que se dan entre los miembros de las familias, antes, durante y después del acto alimenticio. Los hallazgos muestran que aunque las mujeres siguen siendo las mayores responsables de la preparación de alimentos en los hogares, otros miembros del grupo familiar participan en algunas actividades conexas. De igual forma, las dinámicas laborales y de la vida urbana en las grandes ciudades influyen en las prácticas alimentarias (cocinar, lugares y frecuencia de alimentación), dado que los ritmos y horarios del trabajo e incluso la organización interna de las familias y sus transformaciones, son factores que conducen a la desestructuración de la comida como parte de la vida social (Quintero, 2008).

De igual forma, son amplios los estudios que desde el nivel de pregrado y posgrado se han adelantado para conocer el comportamiento alimentario en la vida cotidiana de las familias, focalizando sectores populares urbanos 0 campesinos de las zonas rurales (Barrera, 1997; Barrantes \& Villalobos, 2000; Ramos, 2001; López \& Herrera, 2007). Los análisis revelan las tradiciones alimentarias aprendidas y su transmisión intergeneracional, así como también la introducción de nuevas prácticas o modificación de ciertas creencias respecto a los patrones de alimentación, teniendo en cuenta el contexto social, político, económico y cultural que incide en éstos.

Llama la atención que aunque estos estudios proponen como unidad de análisis a las familias, la recolección de información se centra en las mujeres, lo que evidencia el mantenimiento del sesgo sociocultural que liga mujer y familia como unidades inseparables, más aún en lo que respecta a la alimentación, tradicionalmente delegada como responsabilidad de las mujeres en el hogar. En pocos casos se consideran las familias como organización social a partir de la cual es posible dimensionar la estrecha relación entre la organización social y familiar en sus múltiples dimensiones, así como la expresión de desigualdades sociales en las prácticas de alimentación que tienen lugar en el ámbito doméstico.

\section{LOS APORTES DE LA SOCIOLOGÍA DE LA ALIMENTACIÓN}

La situación de hambre en el mundo ha conducido a un interés creciente en lo relativo a la alimentación, tanto en el campo académico, como en el campo de la acción política, de manera tal que el conocimiento sistematizado sobre las condiciones objetivas y subjetivas de la situación alimentaria de las sociedades, sirva de brújula para la definición y la implementación de políticas orientadas a garantizar el derecho a la alimentación de la población.

Los avances y desarrollos científicos permiten que en la actualidad se disponga de información suficiente sobre la composición de los alimentos, los efectos de los diferentes nutrientes en la salud y la nutrición de la población según características de sexo y edad, las tendencias y cambios del consumo, los sistemas agroalimentarios y de conservación, entre otros; mientras que lo relativo a las prácticas concretas de alimentación, las creencias, los saberes, las tradiciones y costumbres alimentarias en diversos grupos sociales han sido poco exploradas.

Algunas razones para ello pueden deberse a la tendencia de considerar la alimentación como un objeto de estudio propio de las ciencias de la salud, de ahí 
el carácter marginal que este ocupa en el análisis de las ciencias sociales y a que la dimensión social de la nutrición recién alcanza auge en las últimas tres décadas, en comparación con otras tendencias de análisis, como se ha señalado anteriormente (Sanz, 2008). Adicionalmente, los rituales, las simbolizaciones, los valores sociales y estéticos expresados en el proceso alimentario han contado con escasos desarrollos, pese a que existe una línea de investigación desde la sociología de la alimentación y la sociología de la comida (Simmel, 1986), impulsada en el contexto europeo, principalmente durante los últimos años.

\section{Qué pretende y qué nos puede aportar}

Aunque la alimentación es un hecho social presente en todas las sociedades y en todas las culturas, los sistemas de producción, significación, clasificación, preparación y consumo de los alimentos cambian de acuerdo con el período histórico de cada sociedad, su relación con el medio ambiente y las prácticas culturales y de ordenamiento social en el que se insertan.

Desde una mirada sociológica, se busca reconocer la alimentación como práctica social que contiene una dimensión simbólica común a diferentes grupos sociales a los que el individuo pertenece. Interesa comprender la función social de los alimentos y la alimentación en el marco de relaciones sociales con temporalidad y espacialidad que definen un tipo particular de interacción y construcción de la vida social. Se adentra en los significados que adquiere la comida en la configuración de la vida familiar y de la sociedad, los contenidos normativos, la dinámica de organización de tareas y responsabilidades que configuran la vida cotidiana en torno a la satisfacción de esta necesidad fundamental, las redes familiares y sociales que se entrecruzan en el acto alimentario, los saberes inter generacionales respecto a la comida y las formas de vida de los sujetos, las desigualdades sociales y de género en la realización del trabajo doméstico alimentario.

La sociología de la alimentación pretende modificar la perspectiva teórica y las herramientas analíticas centradas en el consumo, para focalizar en el comportamiento de los sujetos, respecto a intereses y condiciones que intervienen en el acceso, preparación y disposición de los alimentos y en el acto alimentario. Este cambio de visión busca destacar la diversidad y la heterogeneidad de procesos intervinientes en las situaciones alimentarias, generalmente invisibles en los análisis tendientes a la generalización, trascender los abordajes económicos y nutricionales predominantes e incluir aspectos motivacionales y variables de tipo social que puedan explicar la permanencia y los cambios de este comportamiento (Díaz \& Gómez, 2001; Sanz, 2008).

Justamente, la debilidad de marcos analíticos que ofrezcan explicaciones sustantivas sobre las percepciones y gustos que inciden en el comportamiento alimentario, las representaciones sobre la alimentación y su contribución al bienestar, las desigualdades sociales y de género que discurren paralelamente a la comida, las relaciones de poder y su interrelación con la escala de jerarquización social, la vinculación de la comida con la estructura micro y macro de las sociedades, justifican la necesidad de una mirada sociológica al fenómeno alimentario.

En síntesis, una perspectiva sociológica contribuye a develar y destacar los contenidos subjetivos y simbólicos de la alimentación que enmarca el fenómeno. Es una contribución para avanzar en el posicionamiento de los análisis micro sociales que pongan al descubierto la multiplicidad de factores que inciden en la conducta alimentaria, ajenos a la pretensión de homogeneidad, determinación o 
generalización que ha caracterizado las investigaciones en la materia, por parte de otras disciplinas.

\section{Lo que está hecho y lo que queda por hacer}

La diversidad de temáticas y enfoques de la sociología de la alimentación resulta bastante extensa y diferencial según el foco de análisis y el contexto en el que se desarrolle, razón por la cual en este apartado destaco aquellos ámbitos temáticos que pueden ser de utilidad para los estudios de carácter micro interesados en el análisis de la alimentación familiar, considerando el estado de la cuestión que presentan Sanz (2008), Díaz y Gómez (2001) y Pérez y Díez-Urdanivia (2007).

1. Las mujeres y su relación con la salud y la alimentación. Se trata de estudios empíricos en poblaciones concretas, generalmente mujeres gestantes y lactantes en condición de riesgo y vulnerabilidad, interesados en identificar los hábitos en el cuidado de la salud familiar, siendo la alimentación un factor fundamental para el adecuado desarrollo de niños, niñas y personas de la familia (Rotenberg \& De Vargas, 2004; Álvarez \& González 2002; Arana, 1982; Ysunza, 1986).

Esta línea de investigación, impulsada especialmente por los profesionales de la salud y la nutrición, mantiene una visión biologicista sobre la lactancia materna centrada en el recién nacido, su crecimiento y desarrollo y los procesos relativos a la incorporación de alimentos sólidos en los niños menores de un año. La concepción cultural que identifica a las mujeres/madres como las directas responsables del cuidado de los niños y las niñas, explica el porqué son ellas las unidades de información en la mayoría de los casos. Sin embargo, en la búsqueda de explicaciones multicausales, es importante considerar el contexto familiar y social en que se adelanta la lactancia materna y el papel que otros miembros cuidadores en la familia (padre, abuela u otros parientes) juegan en el desarrollo humano de los menores. Adicionalmente, considerar las nuevas teorías sobre la masculinidad, para reivindicar el rol social que cumple el padre en el cuidado de su hijo, más allá de que biológicamente no sea proveedor directo de alimento en los primeros meses.

2. La composición nutricional de los alimentos. La asociación entre desnutrición y pobreza suele ser el enfoque desde el cual se abordan los patrones de consumo alimentario en grupos vulnerables, bien sea por condiciones de pobreza o de desplazamiento (Prada, Herrán \& Ortiz, 2008; Crovetto, 2002), para determinar las características nutricionales del consumo diferenciado por sexo y edad, en niños, niñas y jóvenes; no obstante, los adultos y particularmente las mujeres, suelen estar al margen de este tipo de análisis. Sin embargo, estudios desde la perspectiva de género (Pérez y Díez-Urdanivia, 2007; Vizcarra, 2008; Charles \& Kerr, 1995) demuestran las diferencias en el consumo alimentario de las mujeres, bien sea por la desigual distribución de alimentos al considerar que son los hombres quienes requieren mayor cantidad y calidad de alimentos o porque la atención a los demás miembros de la familia dejan de lado su autocuidado. Profundizar en las concepciones culturales que sostienen las desigualdades de género dentro y fuera del grupo familiar, permitirá entender algunas de las características de la transmisión intergeneracional de la privación, la vulnerabilidad y la feminización de la pobreza.

3. Los desórdenes en relación con la comida. Los nuevos estilos de vida impuestos por la sociedad de consumo han conducido a desórdenes alimentarios como la anorexia, la bulimia o la obesidad, que demandan investigaciones que más allá de la mirada biológica y psicológica, permitan comprender la dimensión social de estos trastornos. Al respecto, han aparecido nuevas narrativas sobre 
comida, cuerpo y género (Gracia \& Comellas, 2007) para auscultar sobre la imagen del cuerpo y las relaciones de poder y discriminación que viven las personas en diversos espacios; no obstante, aún falta mucho por descubrir en esta línea teórica.

4. El espacio público de la comida. Este tipo de estudios se adentran en el mundo de simbolizaciones y rituales de la comida como objeto de consumo y distinción social (Simmel, 1986; Bordieu, 1998; Contreras, 1995). Se analizan los espacios institucionales donde se satisface la necesidad de alimentación: restaurantes, colegios, centros de trabajo, eventos sociales, banquetes, así como también las estructuras culinarias, normas y reglas en la preparación y disposición de los alimentos, como actividad que recrea y atraviesa el conjunto de la vida social. No obstante, son pocos los estudios que se adentran en el conocimiento de las prácticas alimentarias al interior de las familias ?acceso, decisión, preparación, distribución de alimentos y recogida de la mesa? que además de dar cuenta de una forma de organización social, expresa diferencias de desigualdad entre los sexos.

\section{UNA APROXIMACIÓN SOCIOLÓGICA A LAS PRÁCTICAS DE ALIMENTACIÓN FAMILIAR}

Analizar las prácticas de alimentación ${ }^{14}$ que desarrollan las familias desde una perspectiva sociológica, constituye un desafío para ahondar en las interrelaciones existentes entre la esfera pública y privada, en la medida que la satisfacción de la necesidad de alimentación es responsabilidad compartida entre el Estado, el mercado, las instituciones y las familias.

Las prácticas cotidianas de alimentación, aunque se realicen en el ámbito privado familiar, hacen parte de relaciones sociales más amplias. En el ámbito público, el papel que el Estado juega en la garantía del derecho a la alimentación mediante programas sociales que transfieren bienes y servicios para proveer recursos alimentarios, la influencia de los sistemas agroalimentarios en la introducción y modificación de patrones de alimentación; la regulación del mercado en la producción, el abastecimiento y el acceso a los alimentos, y el papel que juega la comunidad, los amigos, familiares, entidades sin ánimo de lucro y otro tipo de redes sociales, son factores que inciden en la estructuración de las prácticas de alimentación familiar.

En el ámbito privado, las necesidades nutricionales de los miembros de la familia según diferencias de género, generación, ciclo de vida familiar; la naturaleza del trabajo implicado en esta labor, los aprendizajes y las creencias socioculturales respecto a lo que significa alimentarse, los gustos alimenticios, las relaciones de poder que se producen en la distribución de tareas y responsabilidades en la provisión, compra, preparación, distribución y disposición de los recursos alimentarios, dan cuenta de las formas de organización y distribución del poder entre los sexos, entre generaciones y entre grupos o clases sociales; de las características de producción y consumo de alimentos en una sociedad, y de las formas como se ordenan estos procesos en determinadas sociedades.

Adentrarse en las prácticas de alimentación que se llevan a cabo en el ámbito doméstico familiar, en las relaciones familiares y sociales que se construyen alrededor de la cocina y sus aderezos, se constituye en una oportunidad para desentrañar las características de las relaciones familiares, las tensiones y los conflictos entre los sexos, la organización y el acceso a recursos, las 
desigualdades sociales y las posibilidades de sostenimiento o de cambio de las prácticas y los comportamientos alimentarios, pero también de las relaciones de género en el universo de simbolizaciones y rituales que hacen de la alimentación una posibilidad de realización humana.

\section{BIBLIOGRAFÍA}

- Aguirre, P. (2004). Ricos Flacos y Gordos Pobres, la alimentación en crisis. Buenos Aires: Capital Intelectual.

- Álvarez, U. M. \& González, Z. L. (2002). "Prácticas alimentarias en las familias del área rural de Medellín - Colombia". ALAN [Colombia], 52(1), pp. 55-62.

- Arana, M. (1982). "Las fórmulas lácteas para la alimentación infantil: complementariedad entre las compañías monopólicas y las instituciones estatales". Revista Salud Latinoamericana, pp. 1-6.

- Barrantes, S. M. \& Villalobos, J. (2000). Descripción de los cambios encontrados en los conocimientos, actitudes y prácticas alimentarias de un grupo de personas portadoras de $\mathrm{VIH}$ a partir del diagnóstico, pertenecientes al grupo especial del Hospital Central de la Policía Nacional. Tesis de grado, Facultad de Nutrición y Dietética, Universidad Nacional de Colombia, Bogotá.

- Barrera, O. L. (1997). Comportamiento alimentario en la vida cotidiana de las familias con escolares del sector Serrezuelita, municipio de Funza (Cundinamarca). Tesis de maestría, Facultad de Enfermería, Universidad Nacional de Colombia, Bogotá.

- Bravo, A.; Cass, Y. \& Tranter, D. (2008). "Good food in family day care: Improving nutrition and food safety in family day care". Nutrition \& Dietetics, 65, pp. 47-55, [Dietitians Association of Australia].

- Bejarano, M.; Fuchs, V.; Fernández, N. \& Amancio, O. et al. (2009). "Impacto del acompañamiento familiar sobre la ingestión de alimentos y el estado depresivo de pacientes con cáncer cervicouterino hospitalizadas". Revista Nutrición Hospitalaria, 24, pp. 182-186.

- Booker, H.S. (1949). Income tax and family allowances in Britain. Report of the Royal Commission en Population. Pp. 241 - 247.London.

- Bordieu, P. (1998). La Distinción. Madrid: Taurus.

- Carrasco, H. N. (2007). "Desarrollos de la antropología de la alimentación en América Latina: hacia el estudio de los problemas alimentarios contemporáneos". Revista Estudios Sociales, 15(30), pp. 80-101.

- Charles, N. \& Kerr, M. (1995). "Es así porque es así: diferencias de género y edad en el consumo familiar de alimentos". Alimentación y Cultura. pp. 199 - 217. Barcelona: Ciencias Humanas y Sociales, Universidad de Barcelona.

- Contreras, J. (1995). Alimentación y Cultura. Barcelona: Ciencias Humanas y Sociales, Universidad de Barcelona.

- Contreras, J. \& Espeist, E. (2002). "Tercera edad y prácticas alimentarias: entre las autonomías, las ayudas y el cuidado". Revista ENDOXA, Series filosóficas, 15, pp. 135-151. [Madrid. UNED].

- Crovetto, M. M. (2002). "Cambios en la estructura alimentaria y consumo aparente de nutrientes de los hogares del gran Santiago 1988-1997". Revista Chile Nutre, 29(1), pp. 24-32.

- Cuadernos de Ceam. (2004). Derecho a la alimentación en el Brasil de Lula. UnB Universidad de Brasilia. Centro de Estudios Avançado Multidisciplinares, NEAGRI, Núcleo de Estudios Agrarios. Cuaderno IV, No. 13. Maio 2004. 
- Díaz, M. C. \& Gómez, B. C. (2001). "Del consumo alimentario a la Sociología de la alimentación". Revista Distribución y Consumo, noviembre/diciembre, pp. 5-21.

- Drèze, J. \& Sen, A. (1989). "Hunger in the Modern World". Hunger and public action. Clarendon Press, Oxford.

- Gracia, A. M. \& Comellas, J. (2007). No comerás, narrativas sobre comida, cuerpo y géneros. España: Editorial Icaria.

- García, C. M.; Pardío, L. J.; Arroyo, A. \& Fernández, G. V. (2008). "Dinámica familiar y su relación con hábitos alimentarios". Estudios sobre las culturas contemporáneas, XIV(27), pp. 9-46. [Universidad de Colima, México].

- Grignon, C. \& Grignon, C. (1980). "Styles d'alimentacition et gouts populaires". Revue Francaise de sociologie, (XXI), pp. 531-569.

- Harris, M. (1989). Bueno para comer, enigmas de alimentación y cultura. Madrid: Alianza.

- López, O. \& Herrera, L. (2007). "Alimentación entre los campesinos pescadores de Ayapel (Córdoba)". Revista Kogoró, 1, pp. 7-6. [Facultad de Ciencias Sociales y Humanas, Universidad de Antioquia].

- Machado, A. (2003). Ensayos sobre seguridad alimentaria. Bogotá: Red de Desarrollo Rural y Seguridad Alimentaria RESA, Universidad Nacional de Colombia.

- Maluf, R. S. (1998). "Amartya Sen - Inequality Reexamined (Resenha)". Revista de Economía Política, 18 and 2(70).

- Mantilla, Q. A. \& Morales, G. J. (2008). El derecho a la alimentación en Colombia: situaciones, contextos y vacíos. Bogotá: Fundaexpresión y Observatorio de Seguridad Alimentaria y Nutricional, Universidad Nacional de Colombia.

- Martínez, A. (1985). Mesa y cocina en el S XIX. 2 ed. Bogotá: Planeta.

- Mead, M. (1951). El alimento y la familia. Buenos Aires: Editorial Sudamericana Alsina.

- Mellor, J. W. (1988). "Global Food Balances and Food Security". World Development, 16(9).

- Menasche, R.; Marques, F. C. \& Zanetti, C. (2008). "Autoconsumo e segurança alimentar: a agricultura familiar a partir dos saberes e práticas da alimentação". Revista Nutrir, 21, pp. 145-158.

- Montagut, X. \& Dogliotti, F. (2006). Alimentos globalizados, Soberanía alimentaria y comercio justo. Barcelona: Icaria, Antrazyt.

- Mora de Jaramillo, Y. (1985). Alimentación y cultura en el Amazonas. Bogotá: Fondo Cultural Cafetero.

- Moreno, V. \& Galiano, S. (2006). "La comida en familia: algo más que comer juntos". Nutrición Infantil Pediátrica, 64(11), pp. 554-558.

- Morton, L. W.; Bitto, E. A.; Oakland, M. J. \& Sand, M. (2007). "Accessing food resources: Rural and urban patterns of giving and getting food". Journal Agriculture and Human Values [2008], 25, pp. 107-119.

- Osorio G., C. E. (2002). El arte de comer, simbolismo y cultura de la alimentación en Tierradentro. Popayán: Ministerio de Cultura, Convocatoria de becas de investigación 1999.

- Parikh, K. S. (1992). "Inadequate incomes as the main problem of food insecurity". Food Security: Issues and options, 17, 96.

- Patiño, V. (1984). La alimentación en Colombia y los países vecinos. Cali (Colombia): Programa Editorial Universidad del Valle.

- Pereira, P. (1999). O Conceito de Segurança Alimentar: Debates e controvérsias. Río de Janeiro (Brasil): Universidad Federal Rural do Rio de Janeiro, Instituto de Ciências Humanas e Sociais. Textos Cpda. no. 8, Junho. 
- Pérez de Armiño, K.Ñ. (s.f.). Seguridad Alimentaria. [Documento de trabajo].

- Pérez Gil, R. S. \& Díez-Urdanivia, S. (2007). "Estudios sobre alimentación y nutrición en México: una mirada a través del género". Revista de Salud Pública de México, 49(6), pp. 445-453.

- Prada, G. E.; Herrán, O. F. \& Ortiz, R. (2008). "Patrón alimentario y acceso a los alimentos en familias desplazadas en el municipio de Girón, Santander, Colombia". Revista Panamericana de Salud Pública, 23(4), pp. 257-263.

- Poulain, J. \& Proença, R. (2003). "Methodological approaches on the studies of food practices". Revista Nutrição, 16(4), pp. 365-386.

- Quintero, A. D. (2008). "Alimentación familiar: Una mirada a la cotidianidad en un barrio de clase media en Cali (Colombia)". Revista Economía y Sociedad, 14, pp. 60-81. [Universidad del Valle: Facultad de Ciencias Sociales y Humanas].

- Ramos, B. Y. (2001). Cambios en las prácticas alimentarias a nivel generacional (padres -hijos) de un grupo de personas en la zona rural y urbana del municipio de Lejanías (Meta). Tesis de grado. Facultad de Nutrición y Dietética, Universidad Nacional de Colombia, Bogotá.

- Restrepo, M. C. (2008). La alimentación en la vida cotidiana del Colegio Mayor de Nuestra Señora del Rosario, 1776-1990. Centro de Investigaciones, Estudios y Consultoría CIEC. Bogotá: Editorial Universidad del Rosario.

- Rotenberg, S. \& De Vargas, S. (2004). "Práticas alimentares e o cuidado da saúde: da alimentação da criança à alimentação da família". Revista Brasil. Saúde Materna, Infantil, 4(1), pp. 85-94.

- Sanz, P. J. (2008). "Aportaciones de la sociología al estudio de la nutrición humana: una perspectiva científica emergente en España". Revista Nutrición Hospitalaria, 23(6), pp. 531-535.

- Sen, Amartya. (1981). Poverty and Famine: an essay on entitlement and deprivation. Oxford University Press.

- Simmel, G. (1986). "Sociología de la comida". El Individuo y la Libertad. Ensayos de crítica de la cultura. Barcelona: Península.

- Taren, D. L.; Clark, W.; Chernesky, M. \& Quirk, E. (1990). "Weekly Food Servings and Participation in Social Programs among low income families". Journal Public Health Briefs, 80(11).

- Vizcarra, B. I. (2008). "Entre las desigualdades de género: un lugar para las mujeres pobres en la seguridad alimentaria y el combate al hambre". Argumentos (México), 21(57). pp. 141-173.

- Ysunza, A. (1986). "El abandono de la lactancia materna en México. Su causalidad". Revista de investigación clínica (supl 38), pp. 97-102.

1. Este artículo es producto de la revisión de antecedentes teóricos sobre el estudio de la alimentación como un hecho sociocultural, realizada en el marco de la investigación doctoral denominada "La alimentación familiar como trabajo de cuidado no remunerado: Un estudio en familias rurales y urbanas del municipio de Marmato (Caldas)", responsabilidad exclusiva de la autora, la cual se encuentra en la fase de relevamiento empírico de la información. 2. Profesora-investigadora del Departamento Estudios de Familia de la Universidad de Caldas. Estudiante VIII cohorte Doctorado en Ciencias Sociales, FLACSO (Argentina). Correo electrónico: sandra.franco@ucaldas.edu.co. 3. La preocupación por la alimentación surge ante la imposibilidad de acceder a ella. La crisis alimentaria vivida durante los años 1972-1974, condujo a que una gran cantidad de personas en el mundo murieran ?como también ocurre hoy? de 
hambre. Cuando el hambre se convierte en una necesidad sentida por un gran número de población, se constituye en un problema social y político que debe ser atendido por los Estados. Esta situación generó una línea de estudios desde la economía, la antropología y la sociología de la alimentación, lo cual ha permitido consolidar una tradición investigativa en el área. 4. En el campo de la sociología, los estudios sobre la alimentación tienen desarrollos importantes, principalmente en Francia e Inglaterra desde el siglo XIX, mientas que en España solo a partir de la década del 80 del siglo XX se inician estos estudios. Un desarrollo similar siguió la antropología, en donde ha habido un interés por comprender las prácticas y comportamientos alimenticios como forma de expresión cultural y, en la década del 50, con el avance de la antropología de la alimentación adquiere un carácter aplicado. 5. Para una ampliación del tema de la alimentación desde el área de la salud, se sugiere la revista Nutrición Hospitalaria: http://www.nutricionhospitalaria.com/, donde se dispone de una serie de artículos que relacionan la perspectiva nutricional con los componentes sociales y familiares de la alimentación. 6. Encuesta Nacional de Hogares en el caso de América Latina, Encuesta de Presupuestos Familiares y Panel de Consumo del MAPA en el caso Español, Encuesta de Consumidores y Encuesta Nacional de ingresos y gastos en el caso Colombiano; entre

otros.

7. A partir de la segunda mitad del siglo $X X$, aproximadamente, los países de América Latina han legislado en este campo y los gobiernos han incorporado en sus planes de desarrollo programas de ayuda alimentaria para superar la pobreza y el hambre. En el caso colombiano, a lo largo del siglo XX, se han desarrollado diversos programas para enfrentar el problema de alimentación y nutrición de la población, articulado con las áreas de salud, educación y desarrollo rural. A partir del 2005, se institucionaliza la Política de Seguridad Alimentaria y Nutricional SAN (Documento Consejo de Política Económica y Social CONPES, 113, Marzo de 2.008) de obligatorio cumplimiento en los niveles nacional, departamental y municipal. 8. Las Cumbres Mundiales sobre alimentación, las diversas declaraciones y orientaciones políticas de la FAO, así como la declaración de la ONU sobre los Objetivos de Desarrollo del Milenio para el 2015, constituyen acuerdos multilaterales internacionales que diversos países han firmado para enfrentar el problema del hambre en el mundo. 9. La vulnerabilidad se entiende como la incapacidad que tienen las personas y las familias para satisfacer sus necesidades de bienes y servicios básicos para su calidad de vida, en el mercado. 10. La autora plantea una distinción geopolítica e ideológica presente en los países según la ubicación en los hemisferios; de acuerdo con esto, Asia, América Latina y África conforman los denominados países del sur. 11. El estudio refiere a las prácticas culinarias en Colombia, aunque la autora destaca las limitaciones de la información que podrían restarle representatividad nacional. De un lado, porque la información se concentra en la capital del país, dado el carácter centralista que primaba en la organización sociopolítica, la centralidad en sectores urbanos y la debilidad para ofrecer datos precisos de las particularidades regionales. 12. Los estudios económicos y nutricionales se apoyan en registros oficiales sobre consumo, ingresos y gastos de las familias que realiza el Departamento Nacional de Estadística DANE; la encuesta de la situación alimentaria y nutricional que promueve el Instituto Colombiano de Bienestar Familiar ICBF y el Ministerio de Protección Social y la tabla de composición de alimentos, de manejo y control del Ministerio de Protección Social en todas las entidades de salud. Adicionalmente, se destacan los estudios sobre el problema agrario en Colombia, entre los que se contempla lo relacionado con la producción, abastecimiento y mercado de alimentos, siendo el más destacado el trabajo de Absalón Machado sobre el problema alimentario en Colombia (1986). 
13. La autora indica que el concepto "blanco" se utiliza en la región para denominar a aquellas personas provenientes del interior del país que, generalmente, no suelen pertenecer a comunidades indígenas, característico de la zona amazónica.

14. Las prácticas alimentarias se entienden como el conjunto de actividades cotidianas llevadas a cabo por las familias para satisfacer su necesidad de alimentación. Estas práctica implican un proceso completo que incluye el acceso, almacenamiento, selección, preparación y consumo de alimentos, hasta la recogida de los desechos. 